\title{
USO DE LA AUTOEVALUACIÓN EN ASIGNATURAS RELACIONADAS CON LA DISCAPACIDAD EN ALUMNADO DE EDUCACIÓN
}

\author{
Carolina Fernández-Jiménez \\ María Fernández Cabezas \\ Universidad de Granada \\ Mirian Hervás Torres \\ Universidad de Granada \\ Susana Tallón Rosales \\ Universidad de Huelva \\ M. Tamara Polo Sánchez \\ Universidad de Granada
}

Fecha de Recepción: 19 Marzo 2018

Fecha de Admisión: 10 Abril 2018

\section{RESUMEN}

Se quiso utilizar la autoevaluación como estrategia de evaluación en alumnado de educación. Se analiza la percepción de los estudiantes sobre la adquisición de competencias vinculadas a la discapacidad utilizando como metodología docente el aprendizaje basado en problemas. Los resultados obtenidos permiten concluir que la autoevaluación mediante rúbricas supone una adecuada herramienta para evaluar el grado de adquisición de competencias básicas.

Palabras clave: aprendizaje basado en problemas; autoevaluación; competencia; discapacidad

\section{ABSTRACT}

Application of self-assessment involving Education students enrolled classes on disability.

We used self-assessment as strategies for the assessment of students in the Faculty of Education. We analysed the perception of students regarding their acquisition of skills in the area of disability, using problem-based learning as the teaching methodology. The results suggest that the self-assessment of students using rubrics is an appropriate tool for assessing basic skill levels.

Keywords: problem-based learning; self-assessment; competence; disability

\section{INTRODUCCIÓN}

En los últimos años hemos asistido a la renovación de actividades y metodologías en la educación superior de acuerdo a un nuevo modelo de formación basado en competencias. Este nuevo modelo exige el empleo de metodologías activas, nuevas estrategias didácticas y la evaluación por 


\section{USO DE LA AUTOEVALUACIÓN EN ASIGNATURAS RELACIONADAS CON LA DISCAPACIDAD EN ALUMNADO DE EDUCACIÓN}

competencias que requieren de la interacción con el alumnado, el trabajo en grupo y una evaluación ligada a las tareas de clase. Entre estas técnicas didácticas se ha demostrado que el Aprendizaje Basado en Problemas (ABP) aporta muchos beneficios en la formación universitaria (Arias-Gundín, Fidalgo y García, 2008; Fernández, García, De Caso, Fidalgo y Arias, 2006; Hernández y Lacuesta, 2007), siendo éste además uno de los métodos docentes que más satisfacción produce entre el profesorado (Martínez et al., 2007). Se ha comprobado que el ABP favorece el trabajo en equipo y las relaciones interpersonales, aspectos muy significativos en la educación, por lo que constituye una metodología formativa muy interesante para su aplicación en la formación de futuros profesionales relacionados con este ámbito (Egido et al., 2006). Se ha constatado su eficacia en el desarrollo de competencias en estudiantes de magisterio (Arias-Gundín, Fidalgo, Robledo y Álvarez, 2009; García, Barandiaran, López de Arana, Martínez y Vitoria, 2008), siendo varias las investigaciones realizadas que señalan sus bondades (Alonso-Sanz, 2011; Arias-Gundín, et al., 2009; Egido et al., 2006; García et al., 2008) avalan dichos aspectos. En el enfoque del ABP se fomenta la autonomía cognoscitiva, se enseña y se aprende a partir de problemas que tienen significado para los estudiantes, se utiliza el error como una oportunidad más para aprender y no para castigar y se le otorga una valiosa importancia a la autoevaluación y a la evaluación formativa, cualitativa e individualizada (Dueñas, 2001).

Respecto a las formas de valorar el trabajo del alumnado, la autoevaluación supone una valiosa herramienta al ofrecer información sobre la capacidad de juicio del estudiante acerca de sus avances en el aprendizaje (Fernández, 2010; Ibarra y Rodríguez, 2007). Consiste en la evaluación que lleva a cabo el alumno del propio aprendizaje así como de los factores relacionados con éste, siendo la autoevaluación un objetivo de aprendizaje en sí mismo (Villardón, 2006). Cuando es realizada por los propios estudiantes la autoevaluación parece incrementar el aprendizaje al posibilitar la detección de sus puntos fuertes y débiles en este proceso (Gimeno y Gallego, 2007). Cada vez son más las experiencias en diferentes ámbitos que señalan la conveniencia de su uso (Acedo y RuizCabestre, 2011; Álvarez, 2008; Delgado y Oliver, 2009; Ferrándiz-Vindel, 2011; Moreno, Trigueros y Rivera, 2013; Navarro y González, 2010; Pérez y Urchaga, 2010; Roser, Suriá, Mondragón, Bueno y Rebollo, 2011) y su valor para la evaluación de las competencias actitudinales. La importancia de éstas en asignaturas relacionadas con la discapacidad se ha puesto de manifiesto en diversos trabajos, destacando los realizados en titulaciones relacionadas con la educación (Ferrándiz-Vindel, 2011; Gimeno y Gallego, 2007; Navarro y González, 2010; Tejeiro, et al., 2012). Como en cualquier otro método de evaluación, la elección de los instrumentos a utilizar para la recogida de información resulta fundamental. Las rúbricas o matrices de evaluación constituyen un ejemplo de las herramientas disponibles para que el profesorado pueda llevar a cabo una evaluación formativa (Conde y Pozuelo, 2007). Su diseño y aplicación permiten concretar los criterios con los que valorar las competencias y el aprendizaje, exponiendo al alumnado las expectativas de logro del profesorado respecto a su desempeño (Etxabe, Aranguren y Losada, 2011). Mediante las rúbricas el estudiante puede estimar su propia calificación así como el nivel de competencias adquirido lo que aumenta la motivación y responsabilidad del alumnado sobre su propio proceso de aprendizaje (Villalustre y Del Moral, 2010). Los positivos efectos que estos instrumentos pueden provocar cuando se emplean en la autoevaluación justifican su uso sistemático en el contexto universitario (Rodríguez, Ibarra, Gallego, Gómez y Quesada, 2012). En el presente estudio se quiso utilizar la autoevaluación mediante rúbricas, para determinar el nivel competencial que el alumnado percibe antes y después de la utilización del ABP en materias vinculadas con la discapacidad. 


\section{MÉTODO}

\section{Muestra}

La presente investigación se ha llevado a cabo con alumnado de $2^{0}$ curso matriculado en tres materias que abordaban la atención a la discapacidad en diferentes titulaciones impartidas en la Facultad de Ciencias de la Educación, de la Universidad de Granada. Participaron 229 estudiantes (33 hombres y 196 mujeres) con una media de edad de 22,15 años y desviación típica de 3,760 (Tabla 1).

Tabla 1: Titulaciones, materias implicadas y número de alumnado

\begin{tabular}{ccccc}
\hline & $\begin{array}{c}\text { Educación Social } \\
\text { Materia: } \\
\text { Capacidades } \\
\text { Diversas } \boldsymbol{y} \\
\text { Educación Social }\end{array}$ & $\begin{array}{c}\text { Educación Infantil } \\
\text { Materia: } \\
\text { Trastornos del } \\
\text { Desarrollo }\end{array}$ & $\begin{array}{c}\text { Pedagogía } \\
\text { Materia: } \\
\text { Procesos y Técnicas de } \\
\text { Intervención Psicoeducativa }\end{array}$ & \\
\hline Hombres & 11 & 15 & 7 & Totales \\
Mujeres & 44 & 126 & 26 & 196 \\
$\boldsymbol{N}$ & 55 & 141 & 33 & 229 \\
$\mathbf{\%}$ & 24 & 61,7 & 14,3 & 100 \\
\hline
\end{tabular}

\section{Procedimiento e Instrumentos}

Se seleccionaron un mínimo de competencias que resultaban muy relevantes para el futuro profesional de la educación. Se eligieron a) la capacidad de organización y planificación, competencia que se tiene muy en cuenta a nivel profesional y en las organizaciones, pero poco considerada en la formación universitaria (Marzo, Pedraja y Rivera, 2006); b) la comunicación oral y escrita, habilidades básicas para los profesionales de la educación (Fernández, Torío y Viñuela, 2008), escasamente desarrolladas en los estudiantes universitarios (Arráez, et al., 2008; López-Justicia, Hernández, Fernández, Polo y Chacón, 2008); c) el trabajo en equipo, d) las habilidades interpersonales, competencias consideradas muy relevantes para las organizaciones (Torrelles et al., 2011) y relacionadas con el éxito social y personal aunque escasas en alumnado de educación (Fuentes, González y Raposo, 2008; García de la Vega, 2010; López-Justicia, et al., 2008); y e) el reconocimiento y respeto hacia la discapacidad, competencia fundamental para aquellos profesionales que atienden a este colectivo (López, 2009; Martínez, 2011).

Con el fin de conocer el nivel de logro del alumnado en estas competencias al comenzar el curso, en el mes de octubre y previamente a la aplicación del ABP, se realizó una autoevaluación mediante rúbricas. Posteriormente, al finalizar la materia en el mes de enero, se utilizaron nuevamente las rúbricas para una segunda autoevaluación. Se indicó a los estudiantes que el resultado de la autoevaluación se contemplaría en la nota final representando ésta un $5 \%$ de la calificación final. Posteriormente se llevó a cabo una heteroevaluación por parte del profesor empleando este mismo instrumento, en este caso, formulando los indicadores de la rúbrica en tercera persona.

\section{RESULTADOS}

Para el análisis de los datos se utilizó el paquete estadístico SPSS en su versión 20.

Tras la aplicación del ABP el alumnado considera alcanzar mayor nivel de logro en las competencias reconocimiento de la diversidad, organización y planificación y en menor medida, en el trabajo en equipo (Figura 1). La metodología utilizada no implica, a juicio del alumnado, mejora en su nivel de comunicación oral y escrita, ni tampoco en las habilidades interpersonales. 
Figura 1: Puntuaciones medias de las competencias evaluadas en las materias mediante $A B P$ en los meses de octubre y enero

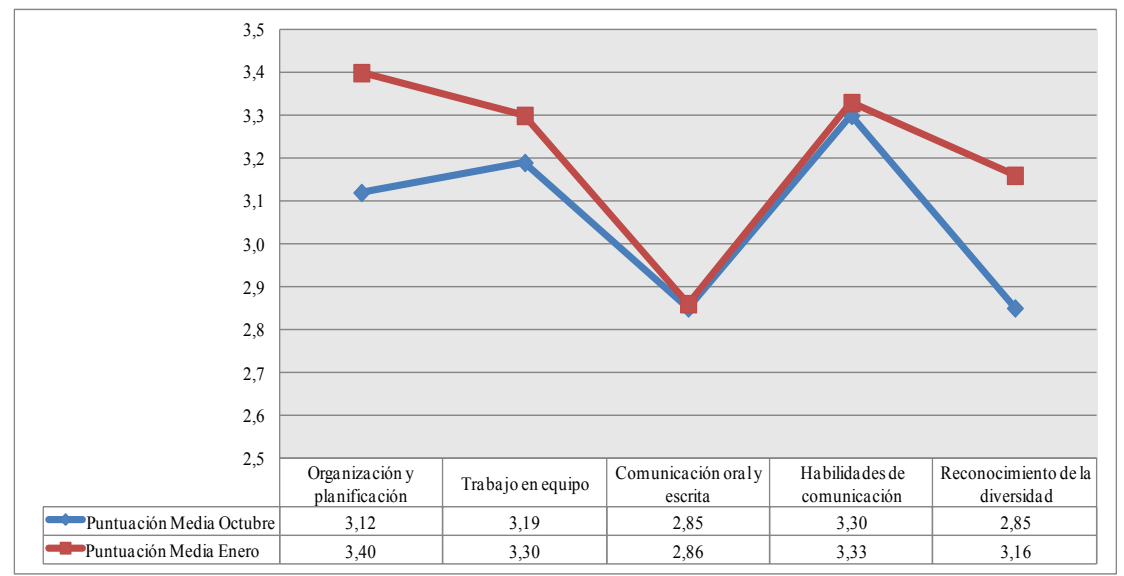

Posteriormente se quiso comparar las puntuaciones medias obtenidas por el alumnado en los descriptores de las competencias evaluadas en los meses de octubre y enero, antes y después de la aplicación del ABP (Figura 2).

Figura 2: Puntuaciones medias en los descriptores de las competencias evaluadas mediante $A B P$ en los meses de octubre y enero

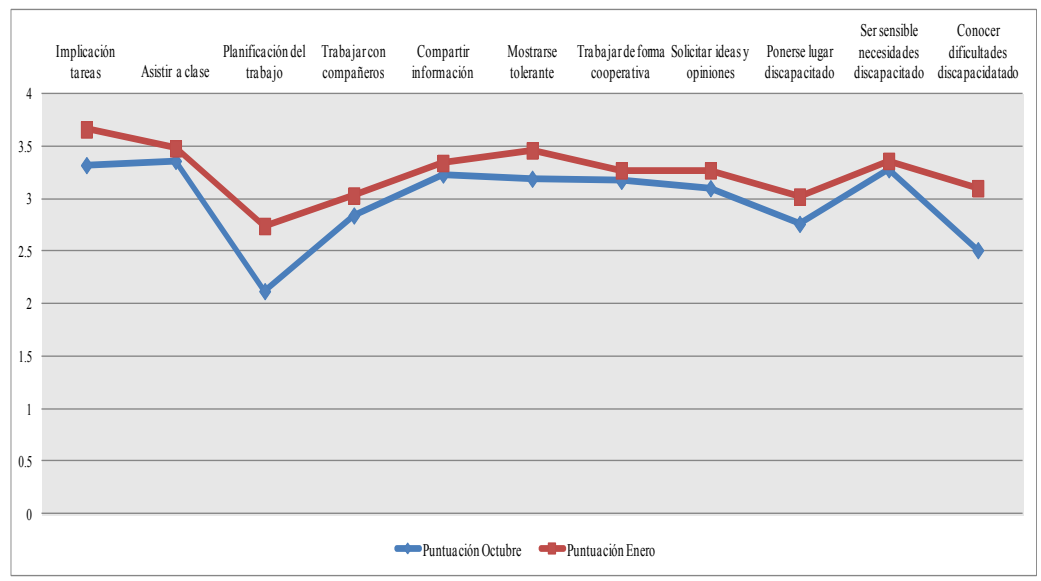

Tras observar diferencias entre ambas evaluaciones se procedió a realizar una prueba t para muestras relacionadas (Tabla 2). 
Tabla 2: Prueba t para muestras relacionadas y tamaño del efecto.

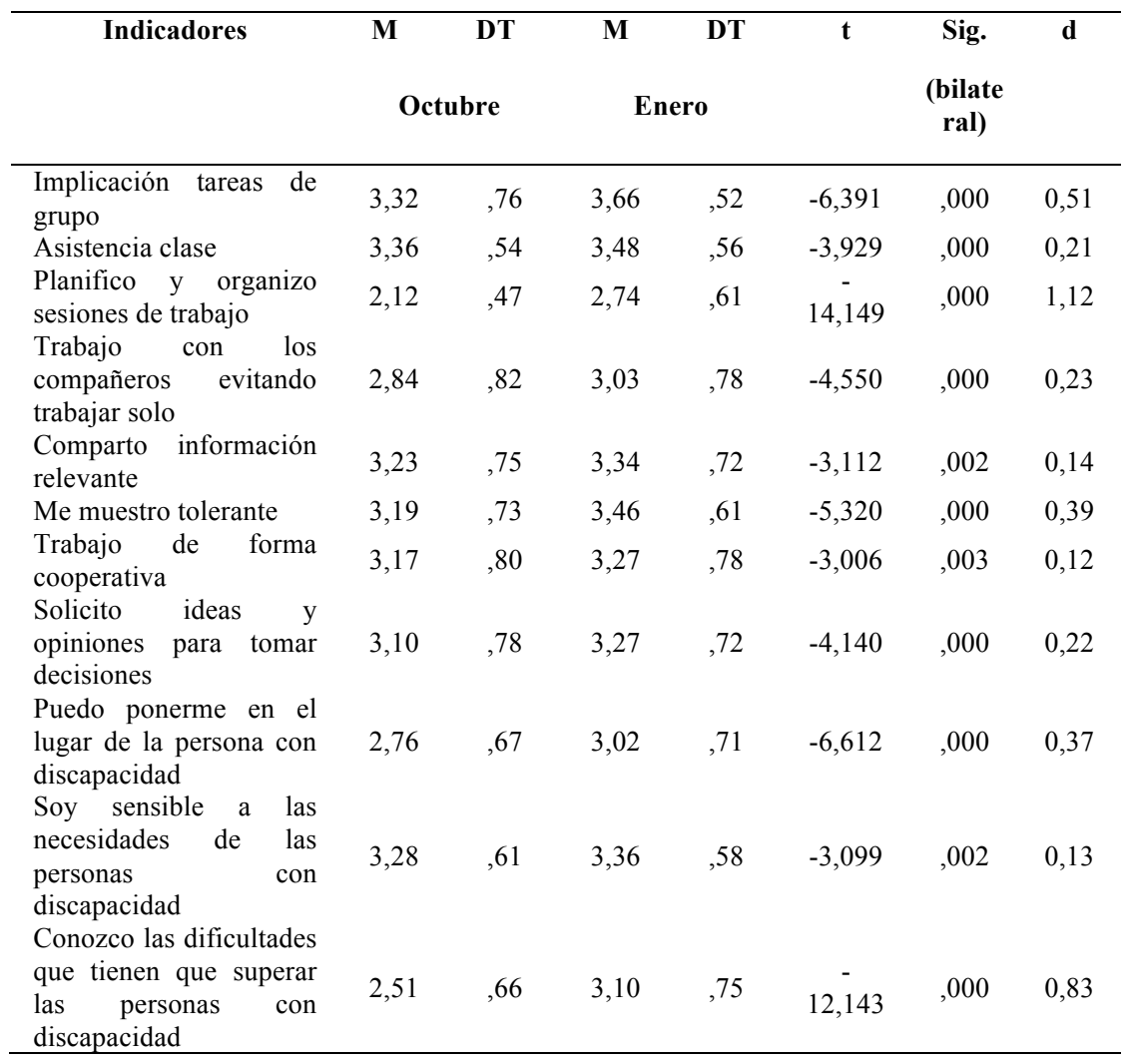

Tras la aplicación de la metodología del ABP, existen diferencias estadísticamente significativas en los descriptores implicación en las tareas de grupo $(t=-6,391, p=, 000)$, asistencia a clase $(t=-$ $3,929, p=, 000)$ y planificación ( $t=14,149, p=, 000)$ correspondientes a la competencia organización. Igualmente, se observan diferencias de logro tras la aplicación del ABP en los siguientes descriptores referidos a la competencia trabajo en equipo: trabajo con los compañeros evitando trabajar solo $(\mathrm{t}=-4,550, \mathrm{p}=, 000)$, comparto información relevante $(\mathrm{t}=-3,112, \mathrm{p}=, 002)$, me muestro tolerante $(\mathrm{t}=$ $5,320, p=, 000)$, trabajo de forma cooperativa $(t=-3,006, p=, 003)$, y solicito ideas y opiniones para tomar decisiones $(t=-4,140 . p=, 000)$. En todos los descriptores referidos a la competencia reconocimiento de la discapacidad se observan diferencias significativas.

\section{DISCUSIÓN}

El estudio descrito en este trabajo propone la autoevaluación como alternativa para valorar la adquisición de competencias por el alumnado empleando el ABP como metodología docente. Los resultados obtenidos parecen indicar que la autoevaluación del alumnado de educación mediante rúbricas permite establecer el nivel de adquisición en las competencias básicas evaluadas. Tal y como señalan Ibarra y Rodríguez (2007) cada vez es más frecuente el empleo de estrategias evaluativas como la autoevaluación, por los beneficios que conlleva para el aprendizaje (Álvarez, 2008; 


\section{USO DE LA AUTOEVALUACIÓN EN ASIGNATURAS RELACIONADAS CON LA DISCAPACIDAD EN ALUMNADO DE EDUCACIÓN}

Delgado y Oliver, 2009; Ferrándiz-Vindel, 2011; Moreno, Trigueros y Rivera, 2013; Navarro y González, 2010; Pérez y Urchaga, 2010; Roser et al., 2011). Esta estrategia de evaluación permite incrementar el aprendizaje al ofrecer un poderoso feedback al estudiante sobre sus avances y limitaciones durante su formación (Gimeno y Gallego, 2007). Además, la autoevaluación se relaciona con un mayor compromiso y autonomía en el proceso educativo, que se refleja en una mayor motivación e interés del alumnado por sus logros (Fernández, 2010).

La utilización de las rúbricas como instrumento de autoevaluación posibilitan al alumnado estimar el nivel de competencias adquirido (Villalustre y Del Moral, 2010). Tras la aplicación del ABP, los estudiantes consideran que mejoran en las competencias reconocimiento de la diversidad y organización y planificación, mientras que no se apreciarían avances en su nivel de comunicación oral y escrita ni tampoco en las habilidades interpersonales. A continuación, se quiso determinar cuáles de los descriptores utilizados para evaluar las competencias mejoraban en opinión del alumnado tras aplicar el ABP. Entendiendo por descriptores los comportamientos observables con los que valorar el nivel de desempeño, la metodología empleada en este estudio parece contribuir a la adquisición y mejora de ciertas conductas y hábitos relacionados con competencias de carácter genérico como son la organización y planificación, el trabajo en equipo y el reconocimiento de la discapacidad. Así, comportamientos referidos a la organización y planificación como la implicación en las tareas, asistir a clase y la planificación o relativos al trabajo en equipo como compartir información relevante, mostrarse tolerante y trabajar de forma cooperativa se han visto favorecidos por la metodología empleada, según el propio alumnado. Similares resultados se han obtenido en otras experiencias de innovación donde estas competencias eran fomentadas mediante metodologías activas (Villalustre y del Moral, 2010). Por otra parte, se constata que ciertos comportamientos relativos a las competencias comunicación oral y escrita y habilidades interpersonales no mejoran con la estrategia docente utilizada. En este sentido, una correcta expresión oral y escrita, la clara transmisión de ideas o la escucha activa no son habilidades que en opinión del alumnado mejoren. Sería importante en este sentido diseñar situaciones problema donde se aprendan y mejoren estas habilidades que, como indican diferentes trabajos en el caso de la expresión oral y escrita, suelen ser deficitarias en universitarios (Arráez et al., 2008; López-Justicia et al., 2008).

\section{REFERENCIAS}

Acedo, M. Á. y Ruiz-Cabestre, F. J. (2011). Una experiencia sobre la evaluación autónoma o participativa: autoevaluación y evaluación por los compañeros. Arbor, 187 (Extra_3), 183-188. doi: 10.3989/arbor.2011.Extra-3n3142.

Alonso-Sanz, A. (2011). Propuesta a estudiantes de Magisterio. Estrategias de diseño de entornos colaborativos desde la Cultura Visual infantil. Arte, Individuo y Sociedad, 23(2),121134.doi:10.5209/rev_ARIS.2011.v23.n2.36259.

Álvarez, I. (2008). La coevaluación como alternativa para mejorar la calidad del aprendizaje de los estudiantes universitarios: valoración de una experiencia. Revista Interuniversitaria de Formación de Profesorado, 22(3), 127-140.

Arias-Gundín, 0., Fidalgo, R., Robledo, P. y Álvarez, L. (2009). Análisis de la efectividad del aprendizaje basado en problemas en el desarrollo de competencias. Actas do X Congresso Internacional Galego-Português de Psicopedagogía. Braga: Universidade do Minho.

Arias-Gundín, 0., Fidalgo, R. y García, J.N. (2008). El desarrollo de las competencias transversales en magisterio mediante el aprendizaje basado en problemas y el método de caso. Revista de Investigación Educativa, 26(2), 431-444.

Arráez, L., Millán, J., Carabantes, D., Lozano, R., Iglesias, I., Palacios, E., Del Castillo, B. y Nogales, 
A. (2008). Adquisición de competencias transversales en alumnos de pregrado de Ciencias de la Salud en la Universidad Complutense: una experiencia positiva. Educación Médica, 11(3), 169-177.

Conde, A. y Pozuelo, F. (2007). Las plantillas de evaluación (rúbrica) como instrumento para la evaluación. Un estudio de caso en el marco de la reforma de la enseñanza universitaria en el EEES. Investigación en la Escuela, 63, 77-90.

Delgado, A.M. y Oliver, R. (2009). Actividades de autoevaluación formativa y aprendizaje autónomo. En J.D. Álvarez, N. Pellín y M.T. Tortosa (Coord.), II Jornadas de Redes de Investigación en Docencia Universitaria: la calidad del proceso universitario desde la perspectiva del cambio (pp.390-401). Alicante: Universidad de Alicante.

Dueñas, V.H. (2001). El aprendizaje basado en problemas como enfoque pedagógico en la educación de la salud. Colombia Médica, 32(4), 189-196.

Egido, I., Aranda, R., Cerrillo, R., De la Herrán, A., De Miguel, S., Gómez, M., Hernández, R., Izuzquiza, D., Murillo, F. J. y Pérez, M. (2006). Aprendizaje basado en problemas (ABP). Estrategia metodológica y organizativa del currículum para la calidad de la enseñanza en los estudios de Magisterio. Revista Interuniversitaria de Formación de Profesorado, 20(3),137-149.

Etxabe, J., Aranguren, K. y Losada, D. (2011). Diseño de rúbricas en la formación inicial de maestros/as. Revista de Formación e Innovación Educativa Universitaria, 4(3), 156-169.

Fernández, A. (2010). La evaluación orientada al aprendizaje en un modelo de formación por competencias en la educación universitaria. Revista de Docencia Universitaria, 8(1), 11-34.

Fernández, M., García, J.N., De Caso, A., Fidalgo, R. y Arias, 0. (2006). El aprendizaje basado en problemas: revisión de estudios empíricos internacionales. Revista de Educación, 341, 397-418.

Fernández, C.M., Torío, S. y Viñuela, Mํ.. P. (2008).La comunicación oral como competencia transversal de los estudiantes de pedagogía y magisterio: presentación del diseño metodológico de una innovación para su trabajo en el aula. Revista de enseñanza universitaria, 31, 26-38.

Ferrándiz-Vindel, A. (2011). La autoevaluación de las competencias en la educación superior. Revista de Investigación en Ciencias Sociales, 7(2), 7-26.

Fuentes, E., González, M. y Raposo, M. (2008). Desarrollar competencias en la formación inicial de maestros: valoraciones, retos y propuestas. Innovación educativa, 18, 45-68.

García, M., Barandiaran, A., López de Arana, E., Martínez, A. y Vitoria, J. R. (2008). El Aprendizaje basado en problemas en la formación inicial del profesorado: innovando para mejorar. $\mathrm{V}$ Congreso de Docencia Universitaria. Valencia: Universidad Politécnica de Valencia.

García de la Vega, A. (2010). Aprendizaje basado en problemas: aplicaciones a la didáctica de las Ciencias Sociales en la Formación Superior. CIDd: II Congrés Internacional de Didactiques 2010. Girona: Universitat de Girona.

Gimeno, M. y Gallego, S. (2007). La autoevaluación de las competencias básicas del estudiante de Psicología. Revista de Psicodidáctica, 12(1), 7-27.

Hernández, A. y Lacuesta, R. (2007). Aplicación del Aprendizaje Basado en Problemas (PBL) bajo un enfoque multidisciplinar: una experiencia práctica. En J. C. Ayala (Coord.), Conocimiento, innovación y emprendedores: Camino al futuro (pp. 30-43). Rioja: Universidad de La Rioja.

Ibarra, M.S. y Rodríguez, G. (2007). El trabajo colaborativo en las aulas universitarias: reflexiones desde la autoevaluación. Revista de Educación, 344, 355-375.

López-Justicia, M. L., Hernández, C. M., Fernández, C., Polo, M.T. y Chacón, H. (2008). Características formativas y socioafectivas del alumnado de nuevo ingreso en la Universidad. Revista Electrónica de Investigación Psicoeducativa, 14(6), 95-116.

Martínez, M.J. (2011). Formación de maestros, atención educativa a alumnos con plurideficiencia y 
estimulación sensoriomotriz. REIFOP, 14(1), 137-150. Extraído el 20 de noviembre de 2013, de http//www.aufop.com.

Martínez, B., García, J. N., Robledo, P., Díez, C., Álvarez, M. L., Marbán, J. M. y Pacheco, D. I. (2007). Valoración docente de las metodologías activas: un aspecto clave en el proceso de Convergencia. Aula abierta, 35(1), 49-62.

Marzo, M., Pedraja, M. y Rivera, P. (2006). Las competencias profesionales demandadas por las empresas: el caso de los ingenieros. Revista de Educación, 341,643-66.

Moreno, A., Trigueros, C. y Rivera, E. (2013). Percepciones sobre la autoevaluación en la formación de profesores de educción física. Revista Internacional de Medicina y Ciencias de la Actividad Física y el Deporte, 13(52), 719-735. Extraído el 12 de febrero de 2014, de http://cdeporte.rediris.es/revista/revista52/artautoevaluacion418.htm

Navarro, I.J. y González, C. (2010). La autoevaluación y la evaluación entre iguales como estrategia para el desarrollo de competencias profesionales. Una experiencia docente en el Grado de Maestro. Revista de Docencia Universitaria, 8(1),187-200.

Pérez, M.C. y Urchaga, J.D. (2010).Autoevaluación de la adquisición de competencias genéricas en una experiencia piloto de adaptación al EEES. CIDd: II Congrés Internacional de Didactiques 2010. Extraído el 20 de diciembre de 2013, de http://hdl.handle.net/10256/2702

Rodríguez, G., Ibarra, M., Gallego, B., Gómez, M.A. y Quesada, V. (2012). La voz del estudiante en la evaluación del aprendizaje: un camino por recorrer en la universidad. RELIEVE, 18(2). Extraído el 20 de noviembre de 2013, de http://www.uv.es/RELIEVE/v18n1/RELIEVEv18n2_2.htm.

Roser, A.M., Suriá, R., Mondragón, J., Bueno, A. y Rebollo, J. (2011). La autoevaluación del alumnado como herramienta de aprendizaje en disciplinas vinculadas a la intervención psicosocial. En M.T. Tortosa, J.D. Álvarez y N. Pellín, IX Jornadas de Redes de Investigación en Docencia Universitaria. Alicante: Universidad de Alicante.

Tejeiro, R., Gomez-Vallecillo, J., Romero, A., Pelegrina, M., Wallace, A. y Emberley, E. (2012). Summative Self-Assessment in Higher Education: Implications of Its Counting towards the Final Mark. Electronic Journal of Research in Educational Psychology, 10(2), 789-812.

Torrelles, C., Coiduras, J., Isus, S., Carrera, F., París, G. y Cela, J. M. (2011). Competencia de trabajo en equipo: definición y categorización. Revista de Currículum y Formación del profesorado, 15(3), 330-344.

Villalustre, L. y Del Moral, M. E. (2010). E-portafolios y rúbricas de evaluación en rural net. PixelBit: Revista de medios y educación, 37, 93-105.

Villardón, M. L. (2006). Evaluación del aprendizaje para promover el desarrollo de competencias. Educatio siglo XXI, 24. Extraído el 20 de noviembre de 2013, de http://revistas.um.es/index.php/educatio/article/view/153 